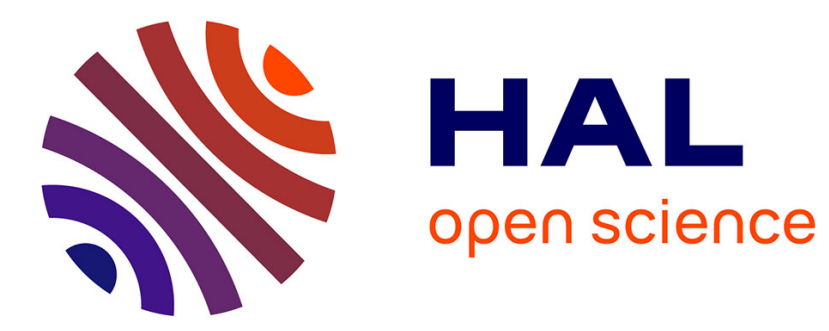

\title{
On the mechanical behavior of fiber-reinforced composites
}

François Hild, Alain Burr, Pascal Feillard

\section{To cite this version:}

François Hild, Alain Burr, Pascal Feillard. On the mechanical behavior of fiber-reinforced composites. Composite Structures, 1997, 39 (3-4), pp.273-282. 10.1016/S0263-8223(97)00119-0 . hal-02342785

\section{HAL Id: hal-02342785 \\ https://hal.science/hal-02342785}

Submitted on 4 Nov 2019

HAL is a multi-disciplinary open access archive for the deposit and dissemination of scientific research documents, whether they are published or not. The documents may come from teaching and research institutions in France or abroad, or from public or private research centers.
L'archive ouverte pluridisciplinaire HAL, est destinée au dépôt et à la diffusion de documents scientifiques de niveau recherche, publiés ou non, émanant des établissements d'enseignement et de recherche français ou étrangers, des laboratoires publics ou privés. 


\title{
On the mechanical behavior of fiber-reinforced composites
}

\author{
François Hild, Alain Burr \& Pascal Feillard \\ Laboratoire de Mécanique et Technologie, E.N.S. Cachan/C.N.R.S./Université Paris VI, 61 avenue du Président Wilson, \\ F-94235 Cachan Cedex, France
}

\begin{abstract}
In this paper, the derivation of the state potential is presented to model the mechanical behavior of fiber-reinforced composites. It allows matrixcracking, interfacial debonding and sliding to be accounted for in the framework of Continuum Damage Mechanics. An application is performed on a unidirectional SiC/SiC composite. (c) 1998 Elsevier Science Ltd. All rights reserved.
\end{abstract}

\section{INTRODUCTION}

It is known that the formation of matrix cracks and the subsequent matrix/fiber interface debonding and sliding are the source of the non-linear stress-strain curves observed when loading Ceramic-Matrix Composites (CMCs) at room temperature. Matrix-cracking, debonding and interfacial sliding reduce the tangent Young's modulus, induce inelastic strains and hysteresis loops $[1,2]$. It is proposed to study these mechanisms in the framework of Continuum Damage Mechanics and to derive constitutive laws suited for structural applications. In this connection an attempt is made to base the formulation on the results of studies of the microstructural mechanisms. These studies are supported by detailed and complex experiments which are beyond the interest or abilities of those concerned with the properties of mechanical components. Therefore, the approach adopted in this study is to use the results of the micromechanical studies to develop constitutive equations which can be formulated from the results of rather simple mechanical tests.

For simplicity reasons, only experimental data on unidirectional architectures subjected to uniaxial stresses are considered but the means of generalization to multiaxial states of stresses has already been demonstrated elsewhere [3]. Special emphasis is put on the sensitivity of the identified micromechanical parameters.
A Continuum Damage Mechanics formulation applied to fiber-reinforced composites is written within the framework of the Thermodynamics of Irreversible Processes [4,5]. The first step in establishing such a model is to identify the state variables which define the state of the material. The second step is to derive the expression of the state potential $\bar{\psi}$ in terms of the state variables and the third one is to determine the evolution laws of the internal variables. The state potential $\bar{\psi}$ is made up of the sum of two terms: viz. the elastic energy density $\bar{\psi}_{e}$ and the stored energy density $\bar{\psi}_{s}$. The elastic part is directly related to the applied load. The stored energy density is concerned with residual stress fields giving rise to macroscopic strains with no applied load. When the local behavior is elastic, the stored energy density is expressed as the total energy density associated with the residual stress field. Therefore, by considering two elastic steps, the total free energy density can be evaluated following a so-called 'cut and paste' technique introduced by Volterra [6]. This approach will be used to model the mechanical behavior of unidirectional as well as $0 / 90$ layered fiber-reinforced CMCs.

Loading a composite consisting of a brittle matrix supported by stronger fibers, usually causes multiple matrix-cracking which is accompanied by interfacial debonding and sliding. In the following, we assume that the whole matrixcracking process occurs at load levels lower 
than the fiber breakage mechanism, and therefore only the former mechanism will be analyzed. Using the same framework, the analysis of fiber breakage and pull-out can be found in Refs [7] and [8]. The aim of this paper is to derive a state potential and to introduce the relevant internal variables to model matrixcracking, debonding and sliding. In the section 'Microscopic description of the degradation mechanisms', the relevant microscopic quantities are introduced to analyze the degradation mechanisms of CMCs. Based upon the results of this section, the following section, 'Macroscopic description of the degradation mechanisms' deals with a macroscopic description of constitutive laws applicable to CMCs. The section 'Micro-macro relationships for unidirectional composites' is devoted to the relationship between microscopic quantities and internal state variables. In the section 'Application to a unidirectional $\mathrm{SiC} / \mathrm{SiC}$ composite', the previous results are applied to model a unidirectional fiber-reinforced $\mathrm{SiC} / \mathrm{SiC}$ composite.

\section{MICROSCOPIC DESCRIPTION OF THE DEGRADATION MECHANISMS}

The matrix cracks, which are assumed to be perpendicular to the fiber direction, cause a stiffness reduction when the stress is tensile. Furthermore it is the closure of the cracks which indicates the onset of increased stiffness when the specimen is subsequently loaded in compression. In the following, we will use elementary cells of length $2 L$, characterizing the crack spacing, and consisting of two different materials (m) and (f) as shown in Fig. 1 [9], where $E$ is the Young's modulus of the unbroken composite, $E_{\mathrm{m}}$ that of part (m), and $E_{\mathrm{f}}$ that of part (f). There is a matrix crack at the center of the cell. Because the matrix contains initial flaws of random strength, there is a distribution of matrix cracks characterized by a probability density function $F(L)$ depending on the applied stress $\bar{\sigma}$. The presence of matrix cracks implies a potential energy density change $\Delta \bar{\varphi}_{e}[10]$

$$
\Delta \bar{\varphi}_{\mathrm{e}}=\frac{1}{\bar{L}} \int_{0}^{+\infty} \Delta \varphi_{e}(L) L F(L) \mathrm{d} L
$$

with

$$
\bar{L}=\int_{0}^{+\infty} L F(L) \mathrm{d} L
$$

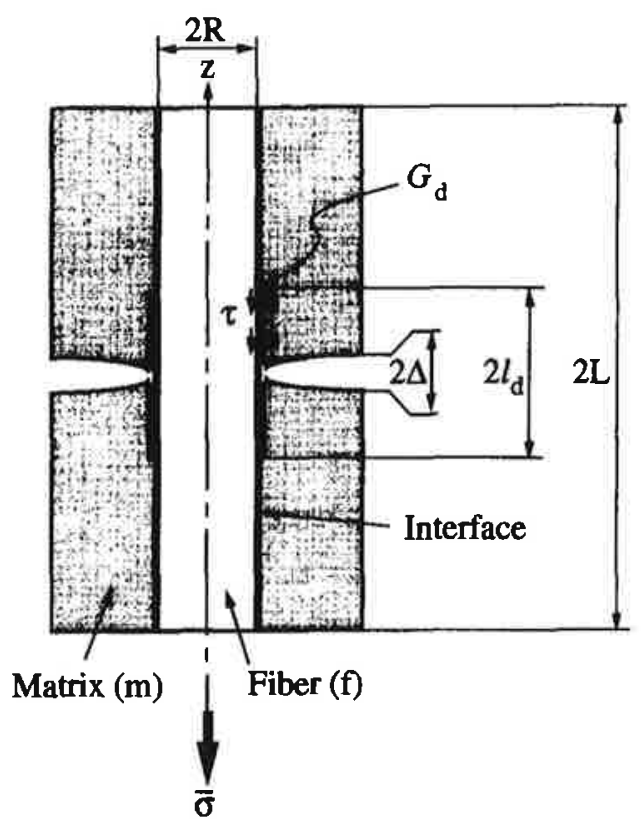

Fig. 1. Elementary cell with crack spacing $2 L$ and debond length $2 I_{d}$.

where $\Delta \varphi_{\mathrm{e}}(L)$ is the average potential energy density change due to the presence of a crack in a cell of length $2 L$, and $2 \bar{L}$ the average crack spacing. In the framework of Linear Elastic Fracture Mechanics, it is known that the potential energy change $\Delta \varphi_{\mathrm{e}}(L)$ is equal to $\bar{\sigma}^{2} \omega_{\mathrm{c}} / E$, where $\omega_{c}$ is dependent on the crack density as well as on the fact that cracks interact or not. This first basic mechanism is purely dissipative and therefore only influences the reversible part of the free energy density $\bar{\psi}_{\mathrm{e}}$.

Debonding followed by sliding gives rise to inelastic strains and hysteresis loops. To describe these phenomena, different models have been proposed $[11,9,12]$. They all consider a friction length $2 l_{F}$ here assumed to be equal to the debond length $2 l_{\mathrm{d}}$ (Fig. 1). Similarly to cracking, which is a mode I mechanism, debonding per se is a purely dissipative mechanism as well. When debonding and slip occur simultaneously, a self-balanced microscopic stress field is involved. The corresponding strains in the matrix and in the fiber are denoted by $\Delta \varepsilon_{\mathrm{m}}(z, L)$ and $\Delta \varepsilon_{\mathrm{f}}(z, L)$, respectively, where $z$ is the current coordinate. These strain differences result from the 'cut and paste' procedure alluded to in the Introduction. By application of the principle of virtual work, the overall inelastic strain $\bar{\varepsilon}_{\text {in }}[13]$ is expressed as

$$
\bar{\varepsilon}_{\mathrm{in}}=\frac{1}{2 \bar{L}} \int_{0}^{+\infty}\left[\int_{-L}^{L} \Delta \varepsilon_{\mathrm{f}}(z, L) \mathrm{d} z\right] F(L) \mathrm{d} L .
$$


Since the additional stress field is selfbalanced the debonding and sliding processes are able to store energy. This result shows that to fully characterize both mechanisms the knowledge of the corresponding stored energy density $\psi_{\text {sd }}$ is crucial. It is expressed as the total elastic energy density associated with the residual stress field due to debonding and sliding

$$
\begin{aligned}
\bar{\psi}_{\mathrm{sd}}= & \frac{1}{2 \bar{L}} \int_{0}^{+\infty}\left[\int _ { - L } ^ { L } \frac { 1 } { 2 } \left\{f E_{\mathrm{f}} \Delta \varepsilon_{\mathrm{f}}^{2}(z, L)\right.\right. \\
& \left.\left.+(1-f) E_{\mathrm{m}} \Delta \varepsilon_{\mathrm{m}}^{2}(z, L)\right\} \mathrm{d} z\right] F(L) \mathrm{d} L
\end{aligned}
$$

where $f$ is the fiber volume fraction. It is worth noting that the expression of the stored energy is $a$ priori only valid if the residual stress field is associated with debonding and sliding. Because of the way these materials are obtained, another residual stress field due to processing is likely to occur. If the residual stress in the matrix is denoted by $\sigma_{\mathrm{pm}}$, the total stored energy density $\bar{\psi}_{\mathrm{s}}$ becomes

$$
\bar{\psi}_{\mathrm{s}}=\bar{\psi}_{\mathrm{sd}}-\bar{\sigma}_{\mathrm{pm}} \bar{\varepsilon}_{\mathrm{in}}+\bar{\psi}_{\mathrm{sp}}
$$

with

$$
\bar{\sigma}_{\mathrm{pm}}=\sigma_{\mathrm{pm}} \frac{E}{E_{\mathrm{m}}} .
$$

The coupling term $-\bar{\sigma}_{\mathrm{pm}} \bar{\varepsilon}_{\text {in }}$ is due to the presence of two residual stresses of different nature. Lastly, the term $\bar{\psi}_{\mathrm{sp}}$ is due to the residual stress field induced by processing. This term is not explicitly given since it is constant. In the following it will be omitted because the free energy density is a potential defined up to an additive constant.

From the present analysis, three different quantities are obtained. A potential energy change due to matrix-cracking, inelastic strains and a corresponding stored energy related to debonding and sliding. Furthermore, a coupling term in the stored energy density is found when the residual stresses due to processing are explicitly accounted for. These results are now used to describe macroscopically the degradation mechanisms.

\section{MACROSCOPIC DESCRIPTION OF THE DEGRADATION MECHANISMS}

The matrix-cracking process described by an internal damage variable $D$ complies with the requirement of a fully dissipative mechanism. In a Continuum Damage Mechanics framework, the presence of a crack results in a stiffness reduction defined by an internal damage variable $D[14,15]$. The Gibbs' elastic energy density of a damaged material is written as [16]

$$
\bar{\varphi}_{\mathrm{e}}(D)=\frac{\bar{\sigma}^{2}}{2 E(1-D)}
$$

so that the potential energy density change can be expressed as

$$
\Delta \bar{\varphi}_{e}=\bar{\varphi}_{e}(D)-\bar{\varphi}_{e}(D=0)=\frac{\bar{\sigma}^{2}}{2 E} \frac{D}{1-D} .
$$

Equation (1) shows that the correct internal variable modeling cracking is the damage variable $D$ whereas the corresponding potential energy density change depends upon the damage variable $D$ as well as the applied stress level $\bar{\sigma}$, and therefore is not an acceptable measure of the degradation due to matrixcracking. Equations (1) and (7) show that the damage variable $D$ is a function of all statistical moments of the crack spacing distribution. A $3 \mathrm{D}$ formulation requires an anisotropic damage description [13]. In the case of cracking perpendicular to the fiber direction, the generalization is straight-forward since the only compliance change is given in the fiber direction, and therefore only one scalar anisotropic damage variable is needed and the present $1 \mathrm{D}$ analysis is still relevant. For a 0/90 layered composite, the Helmholtz free energy density, which is the Legendre transformation of the Gibbs' specific enthalpy, depends upon the damage variables modeling matrix-cracking in the 0 and 90 -degree layers

$$
\bar{\psi}_{\mathrm{e}}=\frac{1}{2} \underline{\varepsilon}: E\left(D_{\mathrm{m}}^{0}, D_{\mathrm{m}}^{90}\right): \underline{\varepsilon}
$$

where $\underline{\varepsilon}$ denotes the total strain tensor, ':' the contraction with respect to two indices, $E$ the elastic stiffness tensor which is a function of the matrix-cracking damage variables $D_{\mathrm{m}}^{\mathrm{o}}$ and $D_{\mathrm{m}}^{90}$ [13].

To fully characterize debonding and sliding two variables are needed. The first one is the inelastic strain $\bar{\varepsilon}_{\mathrm{in}}$, and the second one, denoted by $d$, is introduced to define the stored energy 
density level associated with debonding and sliding alone

$$
\bar{\psi}_{\mathrm{s}}=\frac{E}{2} \frac{\bar{\varepsilon}_{\mathrm{in}}^{2}}{d}-\bar{\sigma}_{\mathrm{pm}} \bar{\varepsilon}_{\mathrm{in}}
$$

This expression can be found in Refs $[17,18]$ to model concrete and rocks for which there is one damage variable modeling the stiffness reduction when the crack is open and sliding in mode II when the same crack is closed. However, in the study of CMCs there is a mode I matrix crack as well as a mode II debond crack (Fig. 1), therefore two damage variables are needed. Furthermore, there is a non-uniform stress field along the debond crack, thus eqn (9) does not necessarily allow to relate the damage variable $d$ to a debond crack density. In a 3D formulation, a second order tensor is needed to model the inelastic strains due to debonding and slip. In the case of a layered 0/90 composite, a first order approximation only requires the in-plane components (i.e. $\bar{\varepsilon}_{\text {in } 11}, \bar{\varepsilon}_{i n 22}$ and $\bar{\varepsilon}_{\text {in } 12}$ ), of the inelastic strain tensor $\bar{\varepsilon}_{i n}$. Since each operative slip system can be integrated separately in terms of energetic contributions, the internal damage variables can be defined separately for each inelastic strain term. Therefore, there are as many debond damage terms as non-vanishing inelastic strain components [13]

$$
\begin{aligned}
\bar{\psi}_{s}= & \frac{E_{11}}{2} \frac{\bar{\varepsilon}_{\mathrm{in} 11}^{2}}{d_{11}}+\frac{E_{22}}{2} \frac{\bar{\varepsilon}_{\mathrm{in} 22}^{2}}{d_{22}} \\
& +\frac{G_{12}}{2} \frac{\bar{\varepsilon}_{\mathrm{in} 12}^{2}}{d_{12}}-\bar{\sigma}_{\mathrm{mp}}: \bar{\varepsilon}_{\mathrm{in}}
\end{aligned}
$$

where $E_{11}, E_{22}$, are the Young's moduli along the 1-direction and the 2-direction, respectively, and $G_{12}$ is the shear modulus in the 1-2 plane. It is worth noting that the residual stress tensor $\bar{\sigma}_{m p}$ is an average tensor in the composite to be computed for each specific architecture. In the case of cracking perpendicular to the fiber direction, only one inelastic strain component is different from zero, viz. the normal component along the fiber direction. Similarly, only one scalar debond damage variable is needed.

Because the residual stresses due to processing are assumed to be constant over the whole composite length, there is no need to introduce another internal variable to describe the coupling term in the expression of the stored energy density $\bar{\psi}_{\mathrm{s}}$. In addition to the total strain $\bar{\varepsilon}$, the total free energy density $\bar{\psi}$ is dependent on three internal variables: one damage variable $D$ modeling matrix-cracking and related to the crack spacing distribution, two variables $\bar{\varepsilon}_{\text {in }}$ and $d$ describing debonding and sliding, and related to the debond length distribution and to the crack opening distribution

$$
\bar{\psi}=\frac{E(1-D)}{2}\left(\bar{\varepsilon}-\bar{\varepsilon}_{\mathrm{in}}\right)^{2}+\frac{E}{2} \frac{\bar{\varepsilon}_{\mathrm{in}}^{2}}{d}-\bar{\sigma}_{\mathrm{pm}} \bar{\varepsilon}_{\mathrm{in}} .
$$

The associated forces are defined as follows

$$
\begin{aligned}
\bar{\sigma} & =\frac{\partial \bar{\psi}}{\partial \bar{\varepsilon}}=E(1-D)\left(\bar{\varepsilon}-\bar{\varepsilon}_{\mathrm{in}}\right) \\
Y & =-\frac{\partial \bar{\psi}}{\partial D}=\frac{\bar{\sigma}^{2}}{2 E(1-D)^{2}} \\
\bar{X} & =-\frac{\partial \bar{\psi}}{\partial \bar{\varepsilon}_{\mathrm{in}}}=\bar{\sigma}+\bar{\sigma}_{\mathrm{pm}}-E \frac{\bar{\varepsilon}_{\mathrm{in}}}{d} \\
y & =-\frac{\partial \bar{\psi}}{\partial d}=\frac{E}{2}\left(\frac{\bar{\varepsilon}_{\mathrm{in}}}{d}\right)^{2} \\
& =\frac{\left(\bar{\sigma}+\bar{\sigma}_{\mathrm{pm}}-\bar{X}\right)^{2}}{2 E}
\end{aligned}
$$

Equation (12) defines the macroscopic stress $\bar{\sigma}$. To properly characterize the overall stressstrain behavior, this result shows that the reversible part of the free energy density is crucial. The following equations define the associated forces to each dissipative mechanism studied previously. Equation (13) defines the energy release rate density $Y$ playing a similar role as the energy release rate $\mathscr{G}$ in Linear Elastic Fracture Mechanics. Combining eqns (12) and (13), one shows that the energy release rate density $Y$ is proportional to the square of the 'effective stress' $\bar{\sigma} /(1-D)$ [16]. Equation [14] defines the back stress $\bar{X}$ related to debonding and sliding. Its exact value depends upon the interfacial behavior. Equation [15] defines the stored energy density $y$ released during debonding and sliding. Since the variable $d$ depends upon the details of the interfacial behavior, the definition of its associated force is also dependent upon the interfacial behavior. The damage variable $d$ and its associated force $y$ are 


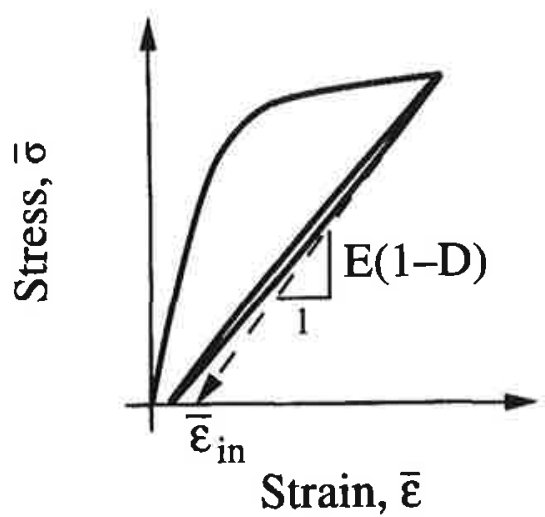

Fig. 2. Schematic stress/strain curve.

not important in terms of overall composite behavior. However in terms of energy contributions these variables are relevant. To determine the evolution laws of the internal variables, two ways can be followed. The first one is using simulations of the complete micromechanical model along the lines developed for instance by Feillard et al. [19] to get the distributions of crack spacings $2 L$ and debond lengths $2 l_{\mathrm{d}}$, and then to compute the state potential. The second one is by performing experiments. The damage variable $D$ is obtained by measuring the initial unloading modulus and the corresponding inelastic strain is $\bar{\varepsilon}_{\text {in }}$ (Fig. 2). To measure the variable $d$, the stored energy $\bar{\psi}_{s}$ has to be evaluated, for instance by using methods developed by Chrysochoos [20] or Cho et al. [21].

For a 0/90 layered composite, the Helmholtz free energy density depends upon the damage variables modeling matrix-cracking in the 0 and 90-degree layers, the inelastic strain tensor as well as the damage variables modeling debonding and sliding

$$
\begin{aligned}
\bar{\psi}= & \frac{1}{2} \underline{\varepsilon}: E\left(D_{\mathrm{m}}^{0}, D_{\mathrm{m}}^{90}\right): \underline{\varepsilon}+\frac{E_{11}}{2} \frac{\bar{\varepsilon}_{\mathrm{in} 11}^{2}}{d_{11}} \\
& +\frac{E_{22}}{2} \frac{\bar{\varepsilon}_{\mathrm{in} 22}^{2}}{d_{22}}+\frac{G_{12}}{2} \frac{\bar{\varepsilon}_{\mathrm{in} 12}^{2}}{d_{12}}-\overline{\bar{\sigma}}_{\mathrm{mp} \cdot \bar{\varepsilon}_{\mathrm{in}}}
\end{aligned}
$$

The associated forces are defined by

$$
\begin{aligned}
& \underline{\sigma}=\frac{\partial \bar{\psi}}{\partial \underline{\varepsilon}} \\
& Y_{\mathrm{m}}^{0}=-\frac{\partial \bar{\psi}}{\partial D_{\mathrm{m}}^{0}}, Y_{\mathrm{m}}^{90}=-\frac{\partial \bar{\psi}}{\partial D_{\mathrm{m}}^{90}}
\end{aligned}
$$

$$
\begin{aligned}
& y_{11}=-\frac{\partial \bar{\psi}}{\partial d_{11}}, y_{22}=-\frac{\partial \bar{\psi}}{\partial d_{22}}, \\
& y_{12}=-\frac{\partial \bar{\psi}}{\partial d_{12}} \\
& \underline{X}=-\frac{\partial \bar{\psi}}{\partial \underline{\varepsilon}_{\text {in }}} .
\end{aligned}
$$

Equations (17)-(20) constitute a generalization of eqns (12)-(15). A description of the identification procedure can be found in Ref. [3].

\section{MICRO-MACRO RELATIONSHIPS FOR UNIDIRECTIONAL COMPOSITES}

In this section, expressions of the three internal variables $D, \bar{\varepsilon}_{\text {in }}$ and $d$ are derived in some particular cases dealing with unidirectional composites. Equation (1) can be written in terms of an equivalent cracking length $\bar{L}_{c}$ defined by

$$
\bar{L} \Delta \bar{\varphi}_{\mathrm{e}}=\bar{L}_{\mathrm{c}} \Delta \varphi_{\mathrm{e}}\left(\bar{L}_{\mathrm{c}}\right) .
$$

It is worth noting that when $\Delta \varphi_{\mathrm{e}}\left(\bar{L}_{\mathrm{c}}\right)$ is inversely proportional to $\bar{L}_{\mathrm{c}}$, then $\Delta \varphi_{\mathrm{e}}(L)$ is inversely proportional to $L$ for any value of $L$ and any distribution $F$. The relevant length to consider is the average crack spacing $\bar{L}$. In that case a local damage theory is applicable since the matrix cracks are not interacting with each other. Otherwise, choosing the equivalent cracking length equal to the average crack spacing is a priori only a zero-th order approximation. The damage variable then depends upon higher order moments of the crack spacing distribution and a non-local damage description seems more appropriate.

Using a shear lag analysis [22], eqns (7) and (21) allow us to link the damage variable $D$ to crack spacings $\bar{L}_{\mathrm{c}}$ and $\bar{L}$ by [10]

$$
\frac{D}{1-D}=\frac{(1-f) E_{\mathrm{m}}}{f E_{\mathrm{f}}} \frac{\tanh \left(\beta \bar{L}_{c}\right)}{\beta \bar{L}}
$$

where the constant $\beta$ is a function of the elastic and geometric properties of the fiber and the matrix. When $\beta \vec{L}_{\mathrm{c}}>2.5$ (i.e. $\tanh \left(\beta \vec{L}_{\mathrm{c}}\right) \cong 1$ ), $D /(1-D)$ is inversely proportional to $\beta \bar{L}$. This last result can also be found in the framework 
of Linear Elastic Fracture Mechanics for non interacting cracks.

The strain distribution $\Delta \varepsilon_{\mathrm{f}}(z, L)$ in the friction zone in the part (f) is assumed to be linear and characterized by a constant interfacial sliding strength $\tau_{0}[23,11]$. The inelastic strain $\bar{\varepsilon}_{i n}$ is given by [12]

$$
\bar{\varepsilon}_{\mathrm{in}}=\frac{\tau \bar{l}_{d}^{2}}{E_{\mathrm{f}} R \bar{L}_{\mathrm{c}}}\left(1+\frac{\sigma_{\mathrm{d}} R}{\tau_{\mathrm{o}} \bar{l}_{\mathrm{d}}}\right)
$$

where $R$ is the fiber radius, and $\bar{l}_{\mathrm{d}}$ is the equivalent debond length defined the same way as the equivalent cracking length $\bar{L}_{\mathrm{c}}$. The debond strength $\sigma_{\mathrm{d}}$ is related to the interfacial debond toughness $\mathscr{G}_{\mathrm{d}}$ by

$$
\sigma_{\mathrm{d}}=2 \sqrt{\frac{(1-f) E_{\mathrm{f}} E_{\mathrm{m}} \mathscr{G}_{\mathrm{d}}}{\mathrm{RE}}}
$$

These results are a $1 \mathrm{D}$ simplification of the axisymmetric calculations given in Ref. [12]. Equations (2) and (23) show that the inelastic strain is a function of the crack spacing distribution as well as the debond length distribution. If the crack spacing distribution is such that the smallest spacing is greater than the debond length, there is a unique debond length value. The definition of the variable $d$ is directly obtained from the evolution of the debond length $\bar{l}_{\mathrm{d}}$ as well as its definition in the expression of the stored energy $\bar{\psi}_{\mathrm{s}}$ given in eqn (4). Using the debond strength $\sigma_{d}$ and the interfacial shear strength $\tau_{0}$, the damage variable can be expressed as

$$
\begin{aligned}
d= & \frac{(1-f) E_{\mathrm{m}}}{f E_{\mathrm{f}}} \frac{\bar{l}_{\mathrm{d}}}{\bar{L}_{\mathrm{c}}} \\
& \times\left[1+\frac{1}{3}\left(1+\frac{\sigma_{\mathrm{d}} R}{\tau_{\mathrm{o}} \bar{l}_{\mathrm{d}}}\right)^{-2}\right]^{-1} .
\end{aligned}
$$

The damage variable $d$ is proportional to the size of the slip zone related to the crack spacing length $\bar{L}_{\mathrm{d}} / \bar{L}_{\mathrm{c}}$. When the ratio $\sigma_{\mathrm{d}} R /\left(\tau_{0} \bar{L}_{\mathrm{d}}\right)$ approaches zero, the damage variable $d$ reaches its lower bound. In that case, the material behaves in a low debond energy regime [24]. Conversely, when the ratio $\sigma_{\mathrm{d}} R /\left(\tau_{0} \bar{L}_{\mathrm{d}}\right)$ approaches infinity, the damage variable $d$ attains its upper bound and the material behaves in a high debond energy regime [24]

$$
\frac{3(1-f) E_{\mathrm{m}}}{4 f E_{\mathrm{f}}} \frac{\bar{L}_{\mathrm{d}}}{\bar{L}_{\mathrm{c}}} \leq d \leq \frac{(1-f)_{E_{\mathrm{m}}}}{f E_{\mathrm{f}}} \frac{\bar{L}_{\mathrm{d}}}{\bar{L}_{\mathrm{c}}} .
$$

The present analysis shows that the key parameter discriminating low versus high debond energy regimes is the ratio $\sigma_{\mathrm{d}} R /\left(\tau_{0} \bar{L}_{\mathrm{d}}\right)$ measuring the relative stress influence due to debonding $\left(\sigma_{\mathrm{d}}\right)$ and friction $\left(\tau_{0} \bar{L}_{\mathrm{d}} / R\right)$. If the stress redistribution is more complex [19], the previous results do not apply: the damage variable $d$ is proportional to the ratio $\bar{L}_{\mathrm{d}} \bar{L}_{\mathrm{c}}$ only if the stress field ahead of the debond crack tip is assumed to be unaltered by the presence of sliding and cracking.

\section{APPLICATION TO A UNIDIRECTIONAL SIC/SIC COMPOSITE}

In the following, we will analyze experiments performed on a unidirectional $\mathrm{SiC} / \mathrm{SiC}$ composite [25]. For this material no matrix-cracking saturation is observed. This assumption is consistent with experimental observations of the evolution of the average crack spacing $2 \bar{L}$ (Fig. 3) as well as that of the damage parameter ratio $D /(1-D)$. This fact allows us to make the hypothesis that the average crack spacing $\bar{L}$ is sufficient to characterize matrix-cracking $\left(\bar{L}_{\mathrm{c}}=\bar{L}\right)$. By combining measurements and micromechanics, eqn (22) shows that there is a way of identifying the parameter $\beta$. Furthermore, the evolution of the average crack density

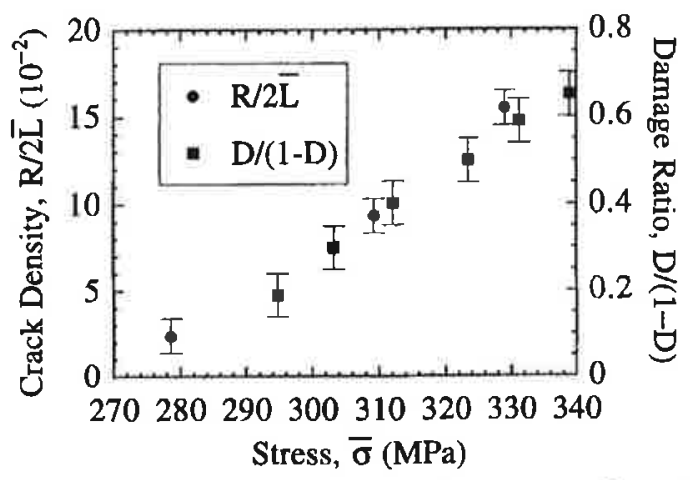

Fig. 3. Evolution of the crack density $R / 2 \bar{L}$ and the damage ratio $D /(1-D)$ as a function of the stress level $\bar{\sigma}$ for a unidirectional $\mathrm{SiC} / \mathrm{SiC}$ composite (after Domergue [25]). 
$R / 2 \bar{L}$ as a function of applied stress is shown to be given by [25]

$$
\frac{R}{\bar{L}}=\frac{R}{\bar{L}_{\mathrm{F}}} \frac{\bar{\sigma}-\bar{\sigma}_{\mathrm{mc}}}{\bar{\sigma}_{\mathrm{F}}-\bar{\sigma}_{\mathrm{mc}}}
$$

where $\bar{\sigma}_{\mathrm{mc}}$ is the macroscopic stress level at which matrix-cracking starts $\left(\bar{\sigma}_{\mathrm{mc}} \cong 275 \mathrm{MPa}\right), \bar{\sigma}_{\mathrm{F}}$ is the failure stress $\left(\bar{\sigma}_{\mathrm{F}} \cong 340 \mathrm{MPa}\right)$, and $2 \bar{L}_{\mathrm{F}}$ is the average crack spacing at failure $\left(2 \bar{L}_{\mathrm{F}} \cong 40 \mu \mathrm{m}\right)$. Equations (22) and (27) can be used to identify the value of $\beta: R \beta=1.09 \pm 0.07$ so that the dimensionless number $\beta \bar{L}_{\mathrm{F}}=2.8$ is in agreement with the hypothesis that $\bar{L}_{\mathrm{c}}=\bar{L}$ (i.e. $\beta \bar{L}_{\mathrm{F}}>2.5$ ).

From a practical stand point, the evolution law can be expressed in a more tractable manner by relating the damage variable $D$ to its associated force $Y$

$$
D=D_{\infty}\left[1-\exp \left\{-\left(\frac{\sqrt{Y}-\sqrt{Y_{\mathrm{mc}}}}{\sqrt{Y_{\mathrm{n}}}}\right)\right\}\right]
$$

where $Y_{\mathrm{n}}$ and $Y_{\mathrm{mc}}$ are material parameters, $Y_{\mathrm{mc}}$ is a threshold value of the energy release rate density below which no damage occurs. At the onset of matrix-cracking, the parameter $Y_{\mathrm{mc}}$ is related to the matrix-cracking stress by (see eqn (13) when $D=0$ )

$$
Y_{\mathrm{mc}}=\frac{\bar{\sigma}_{\mathrm{mc}}^{2}}{2 E} \text {. }
$$

It is worth noting that instead of eqn (28) a more complicated relationship can be obtained between the damage variable $D$ and its associated force $Y$ using eqns (13), (22), (27) and (29). The result of the derivation shows that $\sqrt{Y}$ is the right quantity to consider. Therefore, eqn (28) was written in terms of $\sqrt{Y}$ rather than $Y$ itself. For the unidirectional $\mathrm{SiC} / \mathrm{SiC}$ composite, the following values are identified (Fig. 4)

$$
\begin{aligned}
& D_{\infty}=0.67 \pm 0.05, Y_{\mathrm{mc}}=147 \pm 0.7 \mathrm{kPa}, \\
& Y_{\mathrm{n}}=9.6 \pm 0.4 \mathrm{kPa} .
\end{aligned}
$$

The identified value of $Y_{\mathrm{mc}}$ is in agreement with the value of the matrix-cracking stress $\bar{\sigma}_{\mathrm{mc}}$ according to eqn (29). If eqn (29) is considered (i.e. the value of $Y_{\mathrm{mc}}$ can be obtained from the knowledge of $\bar{\sigma}_{\mathrm{mc}}$ ), the only parameter to identify is $Y_{\mathrm{n}}$, since the value of $D_{\infty}$ is obtained by inspecting eqn (22) when $\bar{L} \rightarrow 0$ and $\bar{L}_{c} \rightarrow 0$, $D \rightarrow D_{\propto}=(1-f) E_{\mathrm{m}} / E$. The value of $D_{\propto}$ is also

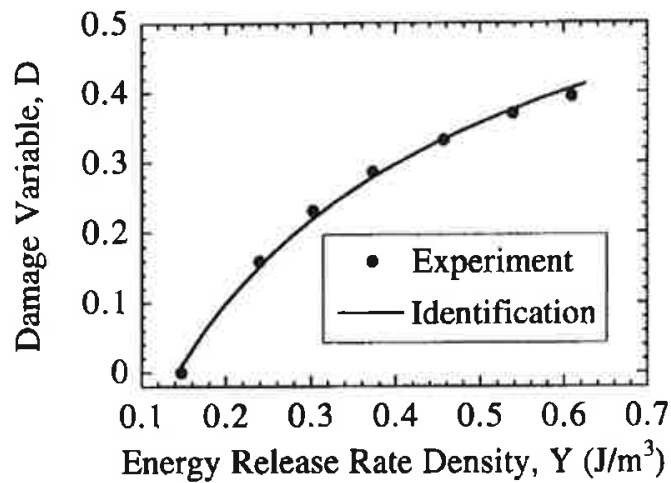

Fig. 4. Evolution of the damage variable $D$ as a function of the energy release rate density $Y$ for a unidirectional $\mathrm{SiC} / \mathrm{SiC}$ composite.

in agreement with that predicted by using the elastic properties of the $\mathrm{SiC} / \mathrm{SiC}$ composite $\left(E_{\mathrm{m}}=300 \mathrm{GPa}, E_{\mathrm{f}}=200 \mathrm{GPa}, f=0.43\right)$.

To completely identify the evolution of the inelastic strains, one needs to determine the residual stress $\bar{\sigma}_{\mathrm{pm}}$, the debond strength $\sigma_{\mathrm{d}}$ and the interfacial shear strength $\tau_{0}$. The difference in coefficients of thermal expansion is assumed to be on the order of $\Delta \alpha \cong 10^{-6} K^{-1}$ and the temperature variation on the order of $\Delta T=1000 \mathrm{~K}$. Consequently, the residual stress level can be approximated by $\bar{\sigma}_{\mathrm{pm}} \cong 80 \mathrm{MPa}$. This value is in reasonable agreement with other studies [25]. In the following, it is assumed that only one debond length is needed to fully characterize the debond regions. The other parameters are obtained by identification of the evolution of the inelastic strains $\bar{\varepsilon}_{\text {in }}$ as a function of the stress level $\bar{\sigma}$

$$
\begin{aligned}
\bar{\varepsilon}_{\mathrm{in}}= & \frac{(1-f)^{2} E_{\mathrm{m}}^{2} R}{4 f^{2} E_{\mathrm{f}} \tau_{0} \bar{L}} \\
& \times\left[\left(\frac{\bar{\sigma}+\bar{\sigma}_{\mathrm{pm}}}{E}\right)^{2}-\left\{\frac{f \sigma_{\mathrm{d}}}{(1-f) E_{\mathrm{m}}}\right\}^{2}\right] .
\end{aligned}
$$

The following values are obtained (see Fig. 5)

$$
\sigma_{d}=550 \pm 10 \mathrm{MPa}, \tau_{0}=46 \pm 5 \mathrm{MPa}
$$

The value of the interfacial shear strength $\tau_{0}$ is in good agreement with other identifications [25] as well as the debond strength (the corresponding interfacial toughness $\mathscr{G}_{\mathrm{d}}$ is on the order of $4 \cdot 2 \pm 0 \cdot 1 \mathrm{~J} / \mathrm{m}^{2}$ ).

If one assumes that the onset of matrix-cracking coincides with the onset of debonding, the value of the debond strength can be easily iden- 


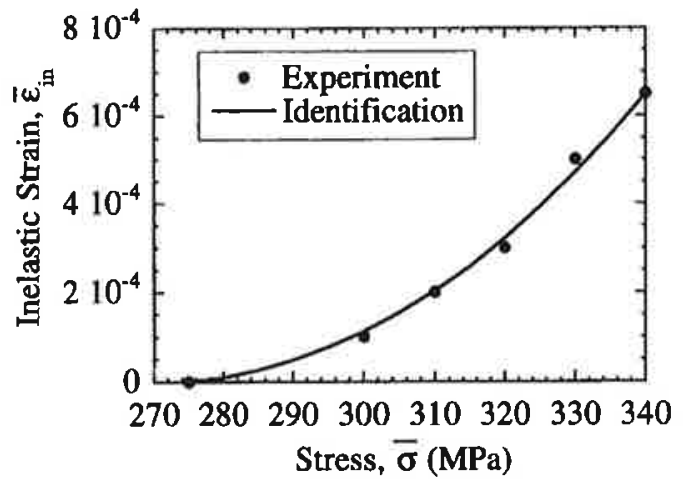

Fig. 5. Evolution of the inelastic strain $\bar{\varepsilon}_{\text {in }}$ as a function of the stress level $\bar{\sigma}$ for a unidirectional $\mathrm{SiC} / \mathrm{SiC}$ composite.

tified, provided that the residual stresses are known

$$
\frac{f \sigma_{\mathrm{d}}}{(1-f) E_{\mathrm{m}}}=\frac{\bar{\sigma}_{\mathrm{mc}}+\bar{\sigma}_{\mathrm{pm}}}{E} .
$$

This hypothesis can be made in the present case. Using eqn (33), a relative variation of the residual stress $\Delta \bar{\sigma}_{\mathrm{pm}} /\left|\bar{\sigma}_{\mathrm{pm}}\right|$ leads to a relative variation of $\Delta \sigma_{\mathrm{d}} / \sigma_{\mathrm{d}}$ expressed as

$$
\frac{\Delta \sigma_{\mathrm{d}}}{\bar{\sigma}_{\mathrm{d}}}=\left(1+\frac{\bar{\sigma}_{\mathrm{mc}}}{\sigma_{\mathrm{d}}}\right)^{-1} \frac{\Delta \bar{\sigma}_{\mathrm{pm}}}{\left|\bar{\sigma}_{\mathrm{pm}}\right|} .
$$

Equation (34) shows that the higher the dimensionless group $\left(1+\bar{\sigma}_{\mathrm{mc}} / \sigma_{\mathrm{d}}\right)$, the lower the sensitivity of the debond strength $\sigma_{\mathrm{d}}$ to the residual stress $\bar{\sigma}_{\mathrm{pm}}$. In the present case eqn (34) becomes

$$
\frac{\Delta \sigma_{\mathrm{d}}}{\bar{\sigma}_{\mathrm{d}}}=0.23 \frac{\Delta \bar{\sigma}_{\mathrm{pm}}}{\left|\bar{\sigma}_{\mathrm{pm}}\right|} .
$$

This result indicates that there is a weak influence of the parameter $\bar{\sigma}_{\mathrm{pm}}$ on the identification of the debond strength $\bar{\sigma}_{\mathrm{d}}$.

Furthermore, by using eqns (27) and (31), the evolution of the inelastic strain can be rewritten as a function of the applied stress $\bar{\sigma}$

$$
\bar{\varepsilon}_{\mathrm{in}}=B \frac{\left(\bar{\sigma}-\bar{\sigma}_{\mathrm{mc}}\right)^{2}\left(\bar{\sigma}+2 \bar{\sigma}_{\mathrm{pm}}+\bar{\sigma}_{\mathrm{mc}}\right)}{E^{2}\left(\bar{\sigma}_{\mathrm{F}}-\bar{\sigma}_{\mathrm{pm}}\right)}
$$

with

$$
B=\frac{D_{\mathrm{F}}}{1-D_{\mathrm{F}}} \frac{(1-f) E_{\mathrm{m}} \beta R}{f \tau_{\mathrm{o}}} .
$$

Equation (37) shows that the identification of $\tau_{0}$ is directly related to the value of $\beta$. There-

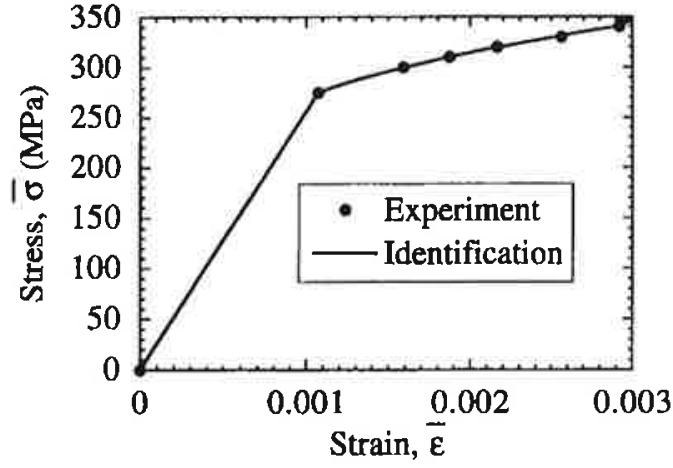

Fig. 6. Evolution of the stress $\bar{\sigma}$ as a function of the strain $\bar{\varepsilon}$ for a unidirectional $\mathrm{SiC} / \mathrm{SiC}$ composite.

fore, a relative variation of the parameter $\beta$, $\Delta \beta / \beta$, leads to the same relative variation of the shear strength $\tau_{0}, \Delta \tau_{0} / \tau_{0}$

$$
\frac{\Delta \tau_{0}}{\tau_{0}}=\frac{\Delta \beta}{\beta} \text {. }
$$

This influence is significantly higher than that exhibited by the residual stress $\bar{\sigma}_{\mathrm{pm}}$ in relation with the debond strength $\bar{\sigma}_{\mathrm{d}}$. These features can be observed when the scatter in values for $\tau_{0}$ and $\varphi_{\mathrm{d}}$ are summarized by Evans et al. [24]. The identified stress/strain curve is compared with the experimental one in Fig. 6: there is a good agreement.

To fully characterize the model, one needs to study the evolution of the damage parameter $d$ defined in eqn (25). Figure 7 shows the evolution of the damage parameter $d$ as a function of stress level $\bar{\sigma}$. The prediction is compared with the upper and lower bounds of the damage parameter given in eqn (26). In the particular case of a unidirectional $\mathrm{SiC} / \mathrm{SiC}$ composite, a

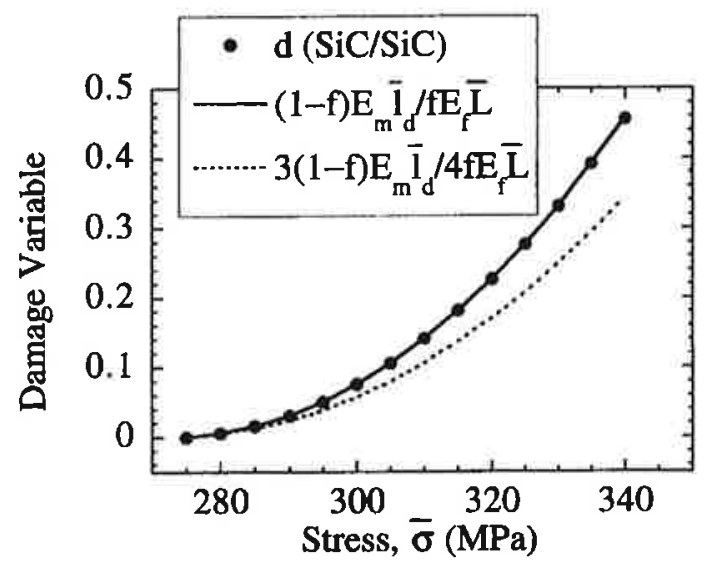

Fig. 7. Evolution of the damage variable $d$ and the corresponding upper bound (solid line) and lower bound (dashed line) as a function of the stress level $\bar{\sigma}$ for a unidirectional $\mathrm{SiC} / \mathrm{SiC}$ composite. 
large debond energy regime is relevant for the whole stress range since the damage parameter coincides with its upper bound. Other composite materials such as layered alumina with carbon/epoxy prepregs are low debond energy materials for which the debond strength is negligible with respect to the average shear stress [26].

\section{CONCLUSIONS}

A Continuum Damage Mechanics formulation has been applied to fiber-reinforced composites. In addition to the total strain, the internal variables which define the state of the material have been identified. Matrix-cracking is described by one damage variable, debonding and sliding are modeled by an inelastic strain and another damage variable measuring the amount of stored energy. These variables are related to microscopic quantities introduced to analyze the degradation mechanisms of CMCs.

Micromechanical parameters are exhibited to model matrix-cracking, interfacial debonding and sliding. Their identification is discussed by analyzing the mechanical behavior of a unidirectional $\mathrm{SiC} / \mathrm{SiC}$ composite. This composite behaves in a large debond energy regime in which the stress levels related to the debond strength are significantly higher than those related to sliding. In the present case, a sensitivity analysis shows that the identification of the interfacial shear strength is more sensitive than that of the debond strength.

The framework presented in this paper can be extended to model layered as well as woven fiber-reinforced composites. The nature of the different internal variables have been discussed. The same formalism can also be used to model high temperature applications in which the change of residual stresses as well as creep mechanisms need to be incorporated. This work is still in progress.

\section{ACKNOWLEDGEMENTS}

This work was partially supported by the Advanced Research Projects Agency through the University Research Initiative, ONR Contract N00014-12-J-1808.

\section{REFERENCES}

1. Beyerley, D., Spearing, S. M., Zok, F. W. and Evans, A. G., Damage, degradation and failure in a unidirectional ceramic-matrix composite. J. Am. Ceram. Soc. 1992, 75, (10), 2719-2725.

2. Pryce, A. W. and Smith, P. A., Modelling of the stress/strain behaviour of unidirectional ceramic matrix composite laminates. J. Mat. Sci. 1992, 27, 2695-2704.

3. Burr, A., Hild, F. \& Leckie, F. A., Continuum description of damage in ceramic-matrix composites Eur. J. Mech. A/Solids, 1997, 16, 53-78.

4. Prigogine, I., Introduction to Thermodynamics of Irreversible Processes. John Wiley and Sons, New York, 1962.

5. Coleman, D. B. and Gurtin, M. E., Thermodynamics with internal variables. J. Chem. Phys. 1967, 47, 597-613.

6. Volterra, V., Sur l'équilibre des corps élastiques multiplement connexes. Annales Scientifiques de l'Ecole Normale Supérieure, Paris 1907, 24, (3), 401-518.

7. Hild, F., Burr, A. and Leckie, F. A., Fiber breakage and fiber pull-out of fiber-reinforced ceramic-matrix composites. Eur. J. Mech. A/Solids 1994, 13, (6), $731-749$.

8. Hild, F., Larsson, P.-L. and Leckie, F. A., Uncoupled and fully coupled approaches to predict macro-initiation in fiber-reinforced ceramic-matrix composites. Comp. Struct. 1994, 29, 365-377.

9. Aveston, J. and Kelly, A., Theory of multiple fracture of fibrous composites. J. Mat. Sci. 1973, 8, 352-362.

10. Burr, A., Feillard, P. \& Hild, F., Identification of a potential to model the behavior of fiber-reinforced composites. C. R. Acad. Sci. Paris, Série IIb, 1996, (t. 323), 377-383.

11. Aveston, J., Cooper, G. A. \& Kelly, A., Single and multiple fracture. Proceedings National Physical Laboratory: Properties of Fiber Composites. IPC Science and Technology Press, Surrey, UK, 1971, pp. 15-26.

12. Hutchinson, J. W. and Jensen, H. M., Models for fiber debonding and fiber pull-out in brittle composites with friction. Mech. Mat. 1990, 9, 139-163.

13. Burr, A., Hild, F. and Leckie, F. A., Micro-mechanics and continuum damage mechanics. Arch. Appl. Mech. $1995,65,(7), 437-456$.

14. Lemaitre, J. \& Dufailly, J., Modélisation et identification de l'endommagement plastique des métaux. Proceedings 3ème congrès français de mécanique. Grenoble, France, 1977.

15. Lemaitre, J. and Dufailly, J., Damage measurements Eng. Fract. Mech. 1987, 28, (5-6), 643-661.

16. Lemaitre, J., $A$ Course on Damage Mechanics. Springer, Berlin, Germany, 1992.

17. Andrieux, S., Un modèle de matériau microfissuré avec frottement. C. R. Acad. Sci. Paris 1981, Série II, (t. 293), 329-332.

18. Andrieux, S., Bamberger, Y. and Marigo, J.-J., Un modèle de matériau microfissuré pour les bétons et les roches. J. Méc. Th. Appl. 1986, 5, (3), 471-513.

19. Feillard, P., Désarmot, G. and Favre, J. P., Theoretical aspects of the fragmentation test. Comp. Sci. Tech. 1994, 50, 265-279.

20. Chrysochoos, A., Maisonneuve, O., Martin, G. and Caumon, H., Plastic and dissipated work and stored energy. Nucl. Eng. Des. 1989, 114, 323-333.

21. Cho, C., Holmes, J. W. and Barber, J. R., Estimation of interfacial shear in ceramic composites from fric- 
tional heating measurements. J. Am. Ceram. Soc. 1991, 74, (11), 2802-2808.

22. Cox, H. L., The elasticity and the strength of paper and other fibrous materials. Br. J. Appl. Phys. 1952, 3, $72-79$.

23. Kelly, A. and Tyson, W. R., Tensile properties of fibre-reinforced metals: copper/tungsten and copper/ molybdenum. J. Mech. Phys. Solids 1965, 13, 329-350.

24. Evans, A. G., Domergue, J.-M. and Vagaggini, E., Methodology for relating the tensile constitutive behavior of ceramic matrix composites to constituent properties. J. Am. Ceram. Soc. 1994, 77, (6), $1425-1435$.

25. Domergue, J.-M., Relationships between the macroscopic behavior of ceramic matrix composites and their constituents properties. Ph.D. Dissertation, University of California, Santa Barbara, 1995.

26. Hild, F., Burr, A. and Leckie, F. A., Matrix cracking and debonding in ceramic-matrix composites. Int. $J$. Solids Struct. 1996, 33, (8), 1209-1220. 